Pacific

Journal of

Mathematics

\title{
Q(N)-GRADED LIE SUPERALGEBRAS ARISING FROM FERMIONIC-BOSONIC REPRESENTATIONS
}

\author{
JIN CHENG
}




\title{
Q(N)-GRADED LIE SUPERALGEBRAS ARISING FROM FERMIONIC-BOSONIC REPRESENTATIONS
}

\author{
JIN CHENG
}

\section{We use fermionic-bosonic representations to obtain a class of $Q(N)$-graded Lie superalgebras coordinatized by quantum tori.}

\section{Introduction}

Root graded Lie algebras were first introduced by Berman and Moody [1992] to understand the generalized intersection matrix algebras of Slodowy. Berman and Moody [1992] classified Lie algebras graded by the root systems of type $A_{l}, D_{l}$, and $E_{6}, E_{7}, E_{8}$ up to central isogeny. Benkart and Zelmanov [1996] classified Lie algebras graded by the root systems of type $B_{n}, C_{n}, F_{4}, G_{2}$ up to central isogeny. Allison et al. [2000] completed the classifications of the above root graded Lie algebras by figuring out explicitly the centers of the universal coverings of those root graded Lie algebras. It turns out that the classification of those root graded Lie algebras played a crucial role in classifying the newly developed extended affine Lie algebras (see [Berman et al. 1996]), which is a generalization of many important Lie algebras, such as affine and toroidal Lie algebras.

Root graded Lie superalgebras are a "super" analog of root graded Lie algebras. Lie superalgebras graded by the root systems of type $A(m, n), B(m, n), C(n)$, $D(m, n)$, and $D(2,1 ; \alpha), F(4), G(3)$ were classified by G. Benkart and A. Elduque. Lie superalgebras graded by the root systems of type $P(N), Q(N)$ were introduced and classified by C. Martínez and E. I. Zelmanov [2003].

Fermionic representations for the affine Kac-Moody Lie algebras were first developed by Frenkel [1980] and Kac and Peterson [1981] independently. Feingold and Frenkel [1985] constructed representations for all classical affine Lie algebras by using Clifford or Weyl algebras with infinitely many generators. Gao [2002] gave bosonic and fermionic representations for the extended affine Lie algebra $\widetilde{\mathrm{gl}} N\left(\mathbb{C}_{q}\right)$, where $\mathbb{C}_{q}$ is the quantum torus in two variables. Chen and Gao [2007] constructed fermionic modules for some $\mathrm{BC}_{N}$-graded Lie algebras, Chen et al. [2006] constructed modules for some $B(0, N)$-graded Lie superalgebras.

Cheng was supported in part by the National Natural Science Foundation of China (No. 11101388). MSC2010: 17B10.

Keywords: root graded Lie superalgebras, quantum tori, fermionic and bosonic representations. 
In this paper, we use fermions and bosons to obtain a class of $Q(N)$-graded Lie superalgebras coordinatized by quantum tori.

The structure of this paper is as follows. In Section 2, we review the definition of $Q(N)$-graded Lie superalgebras and give examples of $Q(N)$-graded Lie superalgebras which coordinatized by quantum tori. In Section 3, we use a tensor product of a fermionic module and a bosonic module to construct the representations for those examples of $Q(N)$-graded Lie superalgebras.

Throughout this paper, we denote the field of complex numbers and the ring of integers by $\mathbb{C}$ and $\mathbb{Z}$ respectively. Let $\mathbb{F}$ be a field of characteristic zero.

\section{Lie superalgebras graded by $Q(N)$}

In this section, we first recall the definition of $Q(N)$-graded Lie superalgebras. Then we construct examples of $Q(N)$-graded Lie superalgebras coordinatized by quantum tori.

Following the notations in [Kac 1977], the finite-dimensional split simple Lie superalgebra $Q(N-1)$ over $\mathbb{F}$ equals $\widetilde{Q}(N-1) / \mathbb{F} I_{2 N}$, where $\widetilde{Q}(N-1)$ consists of the matrices of the form $\left(\begin{array}{cc}a & b \\ b & a\end{array}\right)$, where $a, b \in M_{N}(\mathbb{F})$, and $\operatorname{tr}(b)=0$. Let

$$
\mathcal{H}=\left\{\sum_{i=1}^{N} a_{i}\left(e_{i i}+e_{N+i, N+i}\right) \mid a_{i} \in \mathbb{C}, \sum_{i=1}^{N} a_{i}=0\right\},
$$

then $\mathcal{H}$ is a Cartan subalgebra of $Q(N-1)_{\overline{0}}$.

Define $\varepsilon_{i} \in \mathcal{H}^{*}, i=1, \ldots, N$, by

$$
\varepsilon_{i}\left(\sum_{j=1}^{N} a_{j}\left(e_{j j}+e_{N+j, N+j}\right)\right)=a_{i}
$$

for $i=1, \cdots, N$. Set

$$
Q(N-1)_{\alpha}=\{x \in Q(N-1) \mid[h, x]=\alpha(h) x \text { for all } h \in \mathcal{H}\}
$$

as usual. Then

$$
Q(N-1)=\mathcal{H}+\sum_{\alpha \in \Delta_{\overline{0}}} Q(N-1)_{\overline{0} \alpha}+\sum_{\beta \in \Delta_{\overline{1}}} Q(N-1)_{\overline{1} \beta}
$$

is the root space decomposition of $Q(N-1)$ with respect to the action of $\mathcal{H}$, $\Delta_{Q(N-1)}=\Delta_{\overline{0}} \cup \Delta_{\overline{1}}$, where

$$
\Delta_{\overline{0}}=\Delta_{\overline{1}}=\left\{\varepsilon_{i}-\varepsilon_{j} \mid 1 \leq i \neq j \leq N\right\} .
$$

Definition 2.1 [Martínez and Zelmanov 2003]. A Lie superalgebra $L$ over $\mathbb{F}$ is graded by $Q(N-1)$ if 
( ا) $L$ contains a subsuperalgebra

$$
Q(N-1)=\mathcal{H}+\sum_{\alpha \in \Delta_{Q(N-1)}} Q(N-1)_{\alpha}
$$

(ıl) $L=\sum_{\alpha \in \Delta_{Q(N-1)} \cup\{0\}} L_{\alpha}$;

(lıı) $L_{0}=\sum_{\alpha \in \Delta_{Q(N-1)}}\left[L_{-\alpha}, L_{\alpha}\right]$.

Let $0 \neq q \in \mathbb{C}$. A quantum torus associated to $q$ is the unital associative $\mathbb{C}$-algebra $\mathbb{C}_{q}\left[x^{ \pm 1}, y^{ \pm 1}\right]$ (or simply $\mathbb{C}_{q}$ ) with generators $x^{ \pm}, y^{ \pm}$and relations

$$
x x^{-1}=x^{-1} x=y y^{-1}=y^{-1} y=1 \text { and } y x=q x y .
$$

Let $\operatorname{Matr}_{m, n}\left(\mathbb{C}_{q}\right)$ denote the associative algebra consisting of $m \times n$ matrices with entries in $\mathbb{C}_{q}$.

For two arbitrary positive integers $M$ and $N$ we have an associative superalgebra $\operatorname{Matr}(M, N)\left(\mathbb{C}_{q}\right)$ consisting of $(M, N)$-block matrices with entries in $\mathbb{C}_{q}$, whose $\mathbb{Z}_{2}$-grading is given as follows:

$$
\begin{aligned}
& \operatorname{Matr}(M, N)\left(\mathbb{C}_{q}\right)_{\overline{0}}=\left\{\left(\begin{array}{ll}
A & 0 \\
0 & B
\end{array}\right) \mid A \in \operatorname{Matr}_{M, M}\left(\mathbb{C}_{q}\right), B \in \operatorname{Matr}_{N, N}\left(\mathbb{C}_{q}\right)\right\} \\
& \operatorname{Matr}(M, N)\left(\mathbb{C}_{q}\right)_{\overline{1}}=\left\{\left(\begin{array}{ll}
0 & C \\
D & 0
\end{array}\right) \mid C \in \operatorname{Matr}_{M, N}\left(\mathbb{C}_{q}\right), D \in \operatorname{Matr}_{N, M}\left(\mathbb{C}_{q}\right)\right\} .
\end{aligned}
$$

$\operatorname{Matr}(M, N)\left(\mathbb{C}_{q}\right)$ forms a Lie superalgebra under the supercommutator product $[x, y]:=x y-(-1)^{|x||y|} y x$ for homogeneous $x, y \in \operatorname{Matr}(M, N)\left(\mathbb{C}_{q}\right)$. We denote this Lie superalgebra by $\operatorname{gl}(M, N)\left(\mathbb{C}_{q}\right)$.

Set $\Lambda(q)=\left\{n \in \mathbb{Z} \mid q^{n}=1\right\}$.

We form a central extension of the Lie superalgebra $\operatorname{gl}(M, N)\left(\mathbb{C}_{q}\right)$ as was done in [Gao 2002] and [Chen and Gao 2007]:

$$
\widehat{\operatorname{gl}}(M, N)\left(\mathbb{C}_{q}\right)=\operatorname{gl}(M, N)\left(\mathbb{C}_{q}\right) \oplus\left(\bigoplus_{n \in \Lambda(q)} \mathbb{C} c(n)\right) \oplus \mathbb{C} c_{y}
$$

with Lie superbracket

$$
\begin{aligned}
& {\left[A\left(x^{m} y^{n}\right), B\left(x^{p} y^{s}\right)\right]} \\
& \quad=A\left(x^{m} y^{n}\right) B\left(x^{p} y^{s}\right)-(-1)^{\operatorname{deg} A \operatorname{deg} B} B\left(x^{p} y^{s}\right) A\left(x^{m} y^{n}\right) \\
& \quad+m q^{n p} \operatorname{str}(A B) \delta_{m+p, 0} \delta_{\overline{n+s}, 0} c(n+s)+n q^{n p} \operatorname{str}(A B) \delta_{m+p, 0} \delta_{n+s, 0} c_{y}
\end{aligned}
$$

for $m, p, n, s \in \mathbb{Z}, A, B \in \operatorname{gl}(M, N)_{\alpha}, \alpha=\overline{0}$ or $\overline{1}$, where str is the supertrace of the Lie superalgebra $\operatorname{gl}(M, N), c(u)$ for $u \in \Lambda(q)$ and $c_{y}$ are central elements of $\widehat{\mathrm{gl}}(M, N)\left(\mathbb{C}_{q}\right)$, and $\bar{t} \in \mathbb{Z} / \Lambda(q)$ for $t \in \mathbb{Z}$. 
Let $G=\sqrt{-1}\left(\begin{array}{cc}0 & I_{n} \\ -I_{n} & 0\end{array}\right)$. Using the matrix $G$, we define a $\mathbb{Z}_{2}$-graded subspace $\widetilde{\mathcal{Q}}$ with

$$
\begin{aligned}
& \widetilde{\mathcal{Q}}_{\overline{0}}=\left\{X \in \operatorname{gl}(N, N)\left(\mathbb{C}_{q}\right)_{\overline{0}} \mid X G-G X=0\right\}, \\
& \widetilde{\mathcal{Q}}_{\overline{1}}=\left\{X \in \operatorname{gl}(N, N)\left(\mathbb{C}_{q}\right)_{\overline{1}} \mid X G+G X=0\right\} .
\end{aligned}
$$

Proposition 2.2. The general form of a matrix in $\widetilde{\mathcal{Q}}$ is

$$
\left(\begin{array}{ll}
A & B \\
B & A
\end{array}\right)
$$

where $A, B$ are $N \times N$ submatrices.

As in [Allison et al. 1997], we know that, for the Lie superalgebra $\mathcal{Q}=[\widetilde{\mathcal{Q}}, \widetilde{\mathcal{Q}}]$, we have

$$
\mathcal{Q}=\left\{\left(\begin{array}{ll}
A & B \\
B & A
\end{array}\right) \in \widetilde{\mathcal{Q}} \mid \operatorname{tr}(B) \equiv 0 \bmod \left[\mathbb{C}_{q}, \mathbb{C}_{q}\right]\right\}
$$

Let

$$
\begin{aligned}
& \tilde{g}_{i j}(m, n)=x^{m} y^{n} e_{i j}+x^{m} y^{n} e_{N+i, N+j}, \\
& \tilde{h}_{i j}(m, n)=x^{m} y^{n} e_{i, N+j}+x^{m} y^{n} e_{N+i, j} .
\end{aligned}
$$

Then we have the root space decomposition

where

$$
\mathcal{Q}=\mathcal{Q}_{0} \oplus \bigoplus_{1 \leq i \neq j \leq N} \mathcal{Q}_{\overline{0}\left(\varepsilon_{i}-\varepsilon_{j}\right)} \oplus \bigoplus_{1 \leq i \neq j \leq N} \mathcal{Q}_{\overline{1}\left(\varepsilon_{i}-\varepsilon_{j}\right)},
$$

$$
\begin{aligned}
& \mathcal{Q}_{\overline{0}\left(\varepsilon_{i}-\varepsilon_{j}\right)}=\operatorname{span}_{\mathbb{C}}\left\{\tilde{g}_{i j}(m, n) \mid m, n \in \mathbb{Z}\right\}, \\
& \mathcal{Q}_{\overline{1}\left(\varepsilon_{i}-\varepsilon_{j}\right)}=\operatorname{span}_{\mathbb{C}}\left\{\tilde{h}_{i j}(m, n) \mid m, n \in \mathbb{Z}\right\},
\end{aligned}
$$

and

$$
\begin{aligned}
& \mathcal{Q}_{0}=\operatorname{span}_{\mathbb{C}}\left\{\tilde{g}_{i i}(m, n) \mid 1 \leq i \leq N, m, n \in \mathbb{Z}\right\} \\
& \oplus \operatorname{span}_{\mathbb{C}}\left\{\tilde{h}_{i i}(m, n)-\tilde{h}_{N N}(m, n) \mid 1 \leq i \leq N-1, m, n \in \mathbb{Z}\right\} \\
& \oplus \operatorname{span}_{\mathbb{C}}\left\{\tilde{h}_{N N}(m, n) \mid m, n \in(\mathbb{Z} \times \mathbb{Z}) \backslash(\Lambda(q) \times \Lambda(q))\right\} .
\end{aligned}
$$

As in [Chen and Gao 2007], one easily sees that $\mathcal{Q}$ is a Lie superalgebra graded by $Q(N-1)$. By a direct calculation, we get the central extension of $\mathcal{Q}$ with superbracket as in (2-1) is trivial, and we have:

\section{Proposition 2.3.}

(2-2) $\left[\tilde{g}_{i j}(m, n), \tilde{g}_{k l}(p, t)\right]_{+}=\delta_{j k} q^{n p} \tilde{g}_{i l}(m+p, n+t)-\delta_{i l} q^{t m} \tilde{g}_{k j}(m+p, n+t)$,

(2-3) $\left[\tilde{h}_{i j}(m, n), \tilde{h}_{k l}(p, t)\right]_{+}=\delta_{j k} q^{n p} \tilde{g}_{i l}(m+p, n+t)+\delta_{i l} q^{t m} \tilde{g}_{k j}(m+p, n+t)$,

(2-4) $\left[\tilde{g}_{i j}(m, n), \tilde{h}_{k l}(p, t)\right]_{-}=\delta_{j k} q^{n p} \tilde{h}_{i l}(m+p, n+t)-\delta_{i l} q^{t m} \tilde{h}_{k j}(m+p, n+t)$, for all $m, p, n, t \in \mathbb{Z}$ and $1 \leq i, j, k, l \leq N$. 


\section{Module construction}

Let $\mathcal{R}$ be an arbitrary associative algebra, $\rho= \pm 1$. We define a $\rho$-bracket on $\mathcal{R}$ by

$$
\{a, b\}_{\rho}=a b+\rho b a, \quad a, b \in \mathcal{R} .
$$

Let $\mathfrak{a}$ be the unital associative algebra with $2 N$ generators $a_{i}, a_{i}^{*}, 1 \leq i \leq N$, subject to relations

$$
\left\{a_{i}, a_{j}\right\}_{\rho}=\left\{a_{i}^{*}, a_{j}^{*}\right\}_{\rho}=0
$$

and

$$
\left\{a_{i}, a_{j}^{*}\right\}_{\rho}=\delta_{i j} .
$$

Let the associative algebra $\alpha(N, \rho)$ be generated by

$$
\left\{u(m) \mid u \in \bigoplus_{i=1}^{N}\left(\mathbb{C} a_{i} \oplus \mathbb{C} a_{i}^{*}\right), m \in \mathbb{Z}\right\}
$$

subject to relations

$$
\{u(m), v(n)\}_{\rho}=\{u, v\}_{\rho} \delta_{m+n, 0} .
$$

Then we define the normal ordering as in [Feingold and Frenkel 1985]:

$$
\begin{aligned}
: u(m) v(n): & = \begin{cases}u(m) v(n) & \text { if } n>m, \\
\frac{1}{2}(u(m) v(n)-\rho v(n) u(m)) & \text { if } m=n, \\
-\rho v(n) u(m) & \text { if } m>n,\end{cases} \\
& =-\rho: v(n) u(m):
\end{aligned}
$$

for $n, m \in \mathbb{Z}, u, v \in \mathfrak{a}$. Set

$$
\theta(n)=\left\{\begin{array}{ll}
1 & \text { for } n>0 \\
\frac{1}{2} & \text { for } n=0, \\
0 & \text { for } n<0
\end{array} \quad \text { then } 1-\theta(n)=\theta(-n) .\right.
$$

Then we have

$$
\begin{gathered}
: a_{i}(m) a_{j}(n):=a_{i}(m) a_{j}(n)=-\rho a_{j}(n) a_{i}(m), \\
: a_{i}^{*}(m) a_{j}^{*}(n):=a_{i}^{*}(m) a_{j}^{*}(n)=-\rho a_{j}^{*}(n) a_{i}^{*}(m),
\end{gathered}
$$

and

$$
\begin{aligned}
& a_{i}(m) a_{j}^{*}(n)=: a_{i}(m) a_{j}^{*}(n):+\delta_{i j} \delta_{m+n, 0} \theta(m-n), \\
& a_{j}^{*}(n) a_{i}(m)=: a_{i}(m) a_{j}^{*}(n):-\delta_{i j} \delta_{m+n, 0} \theta(n-m) .
\end{aligned}
$$

Proposition 3.1. In the Clifford algebra $\alpha(N,+1)$ case, the subspaces of quadratic operators are closed under the Lie bracket $[\cdot, \cdot]_{-}$. We have the commutator 
relations

$$
\begin{aligned}
& {\left[a_{i}(m) a_{j}(n), a_{k}(p) a_{l}(t)\right]_{-}=} 0, \\
& {\left[a_{i}(m) a_{j}(n), a_{k}(p) a_{l}^{*}(t)\right]_{-}=-\delta_{i l} \delta_{m,-t} a_{k}(p) a_{j}(n)+\delta_{j l} \delta_{n,-t} a_{k}(p) a_{i}(m), } \\
& {\left[a_{i}(m) a_{j}^{*}(n), a_{k}(p) a_{l}^{*}(t)\right]_{-}=-\delta_{i l} \delta_{m,-t} a_{k}(p) a_{j}^{*}(n)+\delta_{j k} \delta_{n,-p} a_{i}(m) a_{l}^{*}(t), } \\
& {\left[a_{i}(m) a_{j}^{*}(n), a_{k}^{*}(p) a_{l}^{*}(t)\right]_{-}=-\delta_{i l} \delta_{m,-t} a_{k}^{*}(p) a_{j}^{*}(n)-\delta_{i k} \delta_{m,-p} a_{j}^{*}(n) a_{l}^{*}(t), } \\
& \begin{aligned}
{\left[a_{i}^{*}(m) a_{j}^{*}(n), a_{k}^{*}(p) a_{l}^{*}(t)\right]_{-}=0, } \\
{\left[a_{i}(m) a_{j}(n), a_{k}^{*}(p) a_{l}^{*}(t)\right]_{-}=-\delta_{i l} \delta_{m,-t} a_{k}^{*}(p) a_{j}(n)+\delta_{i k} \delta_{m,-p} a_{l}^{*}(t) a_{j}(n) } \\
\quad+\delta_{j k} \delta_{n,-p} a_{i}(m) a_{l}^{*}(t)-\delta_{j l} \delta_{n,-t} a_{i}(m) a_{k}^{*}(p) \\
=\delta_{i l} \delta_{m,-t} a_{j}(n) a_{k}^{*}(p)-\delta_{j l} \delta_{n,-t} a_{i}(m) a_{k}^{*}(p) \\
\quad+\delta_{j k} \delta_{n,-p} a_{i}(m) a_{l}^{*}(t)-\delta_{i k} \delta_{m,-p} a_{j}(n) a_{l}^{*}(t) \\
\quad+\delta_{i k} \delta_{j l} \delta_{m,-p} \delta_{n,-t}-\delta_{i l} \delta_{j k} \delta_{m,-t} \delta_{n,-p} .
\end{aligned}
\end{aligned}
$$

Proposition 3.2. In the Weyl algebra $\alpha(N,-1)$ case, the subspaces of quadratic operators are closed under the Lie bracket $[\cdot, \cdot]_{-}$. We have the commutator relations

$$
\begin{aligned}
{\left[a_{i}(m) a_{j}(n), a_{k}(p) a_{l}(t)\right]_{-} } & =0, \\
{\left[a_{i}(m) a_{j}(n), a_{k}(p) a_{l}^{*}(t)\right]_{-}=} & \delta_{i l} \delta_{m,-t} a_{k}(p) a_{j}(n)+\delta_{j l} \delta_{n,-t} a_{k}(p) a_{i}(m), \\
{\left[a_{i}(m) a_{j}^{*}(n), a_{k}(p) a_{l}^{*}(t)\right]_{-}=} & \delta_{i l} \delta_{m,-t} a_{k}(p) a_{j}^{*}(n)-\delta_{j k} \delta_{n,-p} a_{i}(m) a_{l}^{*}(t), \\
{\left[a_{i}(m) a_{j}^{*}(n), a_{k}^{*}(p) a_{l}^{*}(t)\right]_{-}=} & \delta_{i l} \delta_{m,-t} a_{k}^{*}(p) a_{j}^{*}(n)+\delta_{i k} \delta_{m,-p} a_{j}^{*}(n) a_{l}^{*}(t), \\
{\left[a_{i}^{*}(m) a_{j}^{*}(n), a_{k}^{*}(p) a_{l}^{*}(t)\right]_{-}=} & 0, \\
{\left[a_{i}(m) a_{j}(n), a_{k}^{*}(p) a_{l}^{*}(t)\right]_{-}=} & \delta_{i l} \delta_{m,-t} a_{j}(n) a_{k}^{*}(p)+\delta_{i k} \delta_{m,-p} a_{l}^{*}(t) a_{j}(n) \\
& \quad+\delta_{j l} \delta_{n,-t} a_{i}(m) a_{k}^{*}(p)+\delta_{j k} \delta_{n,-p} a_{l}^{*}(t) a_{i}(m) \\
= & \delta_{i l} \delta_{m,-t} a_{j}(n) a_{k}^{*}(p)+\delta_{j l} \delta_{n,-t} a_{i}(m) a_{k}^{*}(p) \\
& \quad+\delta_{j k} \delta_{n,-p} a_{i}(m) a_{l}^{*}(t)+\delta_{i k} \delta_{m,-p} a_{j}(n) a_{l}^{*}(t) \\
& \quad-\delta_{i k} \delta_{j l} \delta_{m,-p} \delta_{n,-t}-\delta_{i l} \delta_{j k} \delta_{m,-t} \delta_{n,-p} .
\end{aligned}
$$

Remark. The subspaces of fermionic or bosonic quadratic operators are not closed under $[\cdot, \cdot]_{-}$, then we see that the fermionic or bosonic quadratic operators can only correspond to even root vectors.

In the tensor product algebra $\alpha(N,+1) \otimes \alpha(N,-1)$ case, we will identify $u(m) \otimes v(n)=u(m) v(n)$. Then we have

Proposition 3.3. If we express the generators of $\alpha(N,+1)$ and $\alpha(N,-1)$ by $a_{i}(m)$, $a_{j}^{*}(n)$ and $e_{i}(m), e_{j}^{*}(n)$ respectively, we get, for the quadric operators $a_{i}(m) \otimes e_{j}(n)$, 
$a_{i}(m) \otimes e_{j}^{*}(n), a_{i}^{*}(m) \otimes e_{j}(n)$ and $a_{i}^{*}(m) \otimes e_{j}^{*}(n)$, the anticommutation relations

$$
\begin{aligned}
{\left[a_{i}(m) e_{j}(n), a_{k}(p) e_{l}(t)\right]_{+} } & =0 \\
{\left[a_{i}(m) e_{j}(n), a_{k}^{*}(p) e_{l}(t)\right]_{+} } & =\delta_{i k} \delta_{m,-p} e_{j}(n) e_{l}(t), \\
{\left[a_{i}(m) e_{j}(n), a_{k}(p) e_{l}^{*}(t)\right]_{+} } & =\delta_{j l} \delta_{n,-t} a_{i}(m) a_{k}(p), \\
{\left[a_{i}(m) e_{j}(n), a_{k}^{*}(p) e_{l}^{*}(t)\right]_{+} } & =\delta_{i k} \delta_{m,-p} e_{l}^{*}(t) e_{j}(n)+\delta_{j l} \delta_{n,-t} a_{i}(m) a_{k}^{*}(p) \\
& =\delta_{i k} \delta_{m,-p} e_{j}(n) e_{l}^{*}(t)+\delta_{j l} \delta_{n,-t} a_{i}(m) a_{k}^{*}(p) \\
& \quad-\delta_{i k} \delta_{j l} \delta_{m,-p} \delta_{n,-t}, \\
{\left[a_{i}(m) e_{j}^{*}(n), a_{k}(p) e_{l}^{*}(t)\right]_{+} } & =0, \\
{\left[a_{i}(m) e_{j}^{*}(n), a_{k}^{*}(p) e_{l}(t)\right]_{+} } & =\delta_{i k} \delta_{m,-p} e_{l}(t) e_{j}^{*}(n)-\delta_{j l} \delta_{n,-t} a_{i}(m) a_{k}^{*}(p), \\
{\left[a_{i}(m) e_{j}^{*}(n), a_{k}^{*}(p) e_{l}^{*}(t)\right]_{+} } & =\delta_{i k} \delta_{m,-p} e_{j}^{*}(n) e_{l}^{*}(t), \\
{\left[a_{i}^{*}(m) e_{j}(n), a_{k}^{*}(p) e_{l}(t)\right]_{+} } & =0, \\
{\left[a_{i}^{*}(m) e_{j}(n), a_{k}^{*}(p) e_{l}^{*}(t)\right]_{+} } & =\delta_{j l} \delta_{n,-t} a_{i}^{*}(m) a_{k}^{*}(p), \\
{\left[a_{i}^{*}(m) e_{j}^{*}(n), a_{k}^{*}(p) e_{l}^{*}(t)\right]_{+} } & =0 .
\end{aligned}
$$

Proof. We only check (3-4) and (3-5):

$$
\begin{aligned}
& {\left[a_{i}(m) e_{j}(n), a_{k}^{*}(p) e_{l}(t)\right]_{+}=} a_{i}(m) e_{j}(n) a_{k}^{*}(p) e_{l}(t)+a_{k}^{*}(p) e_{l}(t) a_{i}(m) e_{j}(n) \\
&= a_{i}(m) e_{j}(n) a_{k}^{*}(p) e_{l}(t)+\delta_{i k} \delta_{m,-p} e_{j}(n) e_{l}(t) \\
&-a_{i}(m) a_{k}^{*}(p) e_{l}(t) e_{j}(n) \\
&= \delta_{i k} \delta_{m,-p} e_{j}(n) e_{l}(t) ; \\
& {\left[a_{i}(m) e_{j}(n), a_{k}^{*}(p) e_{l}^{*}(t)\right]_{+}=a_{i}(m) e_{j}(n) a_{k}^{*}(p) e_{l}^{*}(t)+a_{k}^{*}(p) e_{l}^{*}(t) a_{i}(m) e_{j}(n) } \\
&=a_{i}(m) e_{j}(n) a_{k}^{*}(p) e_{l}(t)+\delta_{i k} \delta_{m,-p} e_{l}^{*}(t) e_{j}(n) \\
& \quad-a_{i}(m) a_{k}^{*}(p) e_{l}^{*}(t) e_{j}(n) \\
&=a_{i}(m) e_{j}(n) a_{k}^{*}(p) e_{l}^{*}(t)+\delta_{i k} \delta_{m,-p} e_{l}^{*}(t) e_{j}(n) \\
& \quad-a_{i}(m) a_{k}^{*}(p) e_{j}(n) e_{l}^{*}(t)+\delta_{j l} \delta_{n,-t} a_{i}(m) a_{k}^{*}(p) \\
&=\delta_{i k} \delta_{m,-p} e_{j}(n) e_{l}^{*}(t)+\delta_{j l} \delta_{n,-t} a_{i}(m) a_{k}^{*}(p) \\
&-\delta_{i k} \delta_{j l} \delta_{m,-p} \delta_{n,-t} .
\end{aligned}
$$

The proofs of the others are similar.

As in [Feingold and Frenkel 1985; Gao 2002], let $\alpha(N, \rho)^{+}$be the subalgebra generated by $a_{i}(n), a_{j}^{*}(m), a_{k}^{*}(0)$ for $n, m>0$ and $1 \leq i, j, k \leq N$. Let $\alpha(N, \rho)^{-}$ be the subalgebra generated by $a_{i}(n), a_{j}^{*}(m), a_{k}(0)$ for $n, m<0$ and $1 \leq i, j, k \leq N$. Those generators in $\alpha(N, \rho)^{+}$are called annihilation operators while those in $\alpha(N, \rho)^{-}$are called creation operators. Let $V(N, \rho)$ be a simple $\alpha(N, \rho)$-module 
containing an element $v_{0}^{\rho}$, called a"vacuum vector" and satisfying

$$
\alpha(N, \rho)^{+} v_{0}^{\rho}=0 .
$$

So all annihilation operators kill $v_{0}^{\rho}$ and

$$
V(N, \rho)=\alpha(N, \rho)^{-} v_{0}^{\rho} .
$$

The normal orderings of the mixed quadratic elements are given as follows:

$$
\begin{array}{cc}
: a_{i}(m) e_{j}(n):=a_{i}(m) e_{j}(n), \quad: a_{i}(m) e_{j}^{*}(n):=a_{i}(m) e_{j}^{*}(n), \\
: a_{i}^{*}(m) e_{j}(n):=a_{i}^{*}(m) e_{j}(n), \quad: a_{i}^{*}(m) e_{j}^{*}(m):=a_{i}^{*}(m) e_{j}^{*}(m) .
\end{array}
$$

We see that the $\alpha(N,+1) \otimes \alpha(N,-1)$-module

$$
V(N):=V(N,+1) \otimes V(N,-1)=\alpha(N,+1) \otimes \alpha(N,-1) v_{0}^{+} \otimes v_{0}^{-}
$$
is simple.

Motivated by Propositions 2.3, 3.1, 3.2, and 3.3, we let

$$
h_{i j}(m, n)=\sum_{s \in Z} q^{-n s}: a_{i}(m-s) e_{j}(s):+\sum_{s \in Z} q^{-n s}: a_{j}^{*}(s) e_{i}^{*}(m-s):
$$

\section{Lemma 3.4.}

$$
\begin{aligned}
& {\left[h_{i j}(m, n), h_{k l}(p, t)\right]_{+}} \\
& \qquad \delta_{i l} q^{t m} \sum_{s \in Z} q^{-(n+t) s}\left\{: a_{k}(m+p-s) a_{j}^{*}(s):+: e_{j}(s) e_{k}^{*}(m+p-s):\right\} \\
& \quad+\delta_{j k} q^{n p} \sum_{s \in Z} q^{-(n+t) s}\left\{: a_{i}(m+p-s) a_{l}^{*}(s):+: e_{l}(s) e_{i}^{*}(m+p-s):\right\} .
\end{aligned}
$$

Proof. First we have

$$
\begin{aligned}
& {\left[h_{i j}(m, n), h_{k l}(p, t)\right]_{+}} \\
& \quad=\delta_{i l} \sum_{s_{1}, s_{2} \in Z} q^{-n s_{1}-t s_{2}}\left\{\delta_{m-s_{1},-s_{2}} e_{k}^{*}\left(p-s_{2}\right) e_{j}\left(s_{1}\right)+\delta_{m-s_{1},-s_{2}} a_{k}\left(p-s_{2}\right) a_{j}^{*}\left(s_{1}\right)\right\} \\
& \quad+\delta_{j k} \sum_{s_{1}, s_{2} \in Z} q^{-n s_{1}-t s_{2}}\left\{\delta_{s_{1}, s_{2}-p} e_{i}^{*}\left(m-s_{1}\right) e_{l}\left(s_{2}\right)+\delta_{s_{1}, s_{2}-p} a_{i}\left(m-s_{1}\right) a_{l}^{*}\left(s_{2}\right)\right\} .
\end{aligned}
$$

Secondly notice that

$$
e_{k}^{*}\left(p-s_{2}\right) e_{j}\left(s_{1}\right)=e_{j}\left(s_{1}\right) e_{k}^{*}\left(p-s_{2}\right)-\delta_{j k} \delta_{s_{1}, s_{2}-p},
$$

and by the property (3-3) of the normal ordering we have

$$
\begin{aligned}
& a_{k}\left(p-s_{2}\right) a_{j}^{*}\left(s_{1}\right)=: a_{k}\left(p-s_{2}\right) a_{j}^{*}\left(s_{1}\right):+\delta_{j k} \delta_{s_{1}, s_{2}-p} \theta\left(p-s_{2}-s_{1}\right), \\
& e_{j}\left(s_{1}\right) e_{k}^{*}\left(p-s_{2}\right)=: e_{j}\left(s_{1}\right) e_{k}^{*}\left(p-s_{2}\right):+\delta_{j k} \delta_{s_{1}, s_{2}-p} \theta\left(s_{1}+s_{2}-p\right) .
\end{aligned}
$$


Then

$$
\begin{aligned}
e_{k}^{*}\left(p-s_{2}\right) e_{j}\left(s_{1}\right)+a_{k}\left(p-s_{2}\right) a_{j}^{*}\left(s_{1}\right)=: & a_{k}\left(p-s_{2}\right) a_{j}^{*}\left(s_{1}\right):+: e_{j}\left(s_{1}\right) e_{k}^{*}\left(p-s_{2}\right): \\
+ & \delta_{j k} \delta_{s_{1}, s_{2}-p} \theta\left(s_{1}+s_{2}-p\right) \\
& +\delta_{j k} \delta_{s_{1}, s_{2}-p} \theta\left(p-s_{1}-s_{2}\right)-\delta_{j k} \delta_{s_{1}, s_{2}-p} \\
=: & a_{k}\left(p-s_{2}\right) a_{j}^{*}\left(s_{1}\right):+e_{j}\left(s_{1}\right) e_{k}^{*}\left(p-s_{2}\right):
\end{aligned}
$$

since $\theta\left(s_{1}+s_{2}-p\right)+\theta\left(p-s_{1}-s_{2}\right)=1$. We get

$$
\begin{aligned}
& {\left[h_{i j}(m, n), h_{k l}(p, t)\right]_{+}} \\
& =\delta_{i l} \sum_{s_{1}, s_{2} \in Z} q^{-n s_{1}-t s_{2}} \delta_{m-s_{1},-s_{2}}\left\{: a_{k}\left(p-s_{2}\right) a_{j}^{*}\left(s_{1}\right):+: e_{j}\left(s_{1}\right) e_{k}^{*}\left(p-s_{2}\right):\right\} \\
& +\delta_{j k} \sum_{s_{1}, s_{2} \in Z} q^{-n s_{1}-t s_{2}} \delta_{s_{1}, s_{2}-p}\left\{: a_{i}\left(m-s_{1}\right) a_{l}^{*}\left(s_{2}\right):+: e_{i}^{*}\left(m-s_{1}\right) e_{l}\left(s_{2}\right):\right\} \\
& =\delta_{i l} q^{t m} \sum_{s \in Z} q^{-(n+t) s}\left\{: a_{k}(m+p-s) a_{j}^{*}(s):+: e_{j}(s) e_{k}^{*}(m+p-s):\right\} \\
& +\delta_{j k} q^{n p} \sum_{s \in Z} q^{-(n+t) s}\left\{: a_{i}(m+p-s) a_{l}^{*}(s):+: e_{l}(s) e_{i}^{*}(m+p-s):\right\} .
\end{aligned}
$$

Comparing with Proposition 2.3, let

$$
g_{i j}(m, n)=\sum_{s \in Z} q^{-n s}: a_{i}(m-s) a_{j}^{*}(s):+\sum_{s \in Z} q^{-n s}: e_{j}(s) e_{i}^{*}(m-s): .
$$

Then we only need to check the remaining Lie brackets (2-2) and (2-4).

\section{Lemma 3.5.}

$\left[g_{i j}(m, n), h_{k l}(p, t)\right]_{-}=\delta_{j k} q^{n p} h_{i l}(m+p, n+t)-\delta_{i l} q^{t m} h_{k j}(m+p, n+t)$.

Proof. Notice that removing the normal ordering has no effect on Lie bracket; then we have

$$
\begin{aligned}
& {\left[g_{i j}(m, n), h_{k l}(p, t)\right]_{-}=\sum q^{-n s_{1}-t s_{2}}\left[a_{i}\left(m-s_{1}\right) a_{j}^{*}\left(s_{1}\right)+e_{j}\left(s_{1}\right) e_{i}^{*}\left(m-s_{1}\right),\right.} \\
& \left.a_{k}\left(p-s_{2}\right) e_{l}\left(s_{2}\right)+a_{l}^{*}\left(s_{2}\right) e_{k}^{*}\left(p-s_{2}\right)\right]_{-} .
\end{aligned}
$$

Secondly, for $\left[a_{i}\left(m-s_{1}\right) a_{j}^{*}\left(s_{1}\right), a_{k}\left(p-s_{2}\right) e_{l}\left(s_{2}\right)\right]_{-}$we have

$$
\left[a_{i}\left(m-s_{1}\right) a_{j}^{*}\left(s_{1}\right), a_{k}\left(p-s_{2}\right) e_{l}\left(s_{2}\right)\right]_{-}=\delta_{j k} \delta_{s_{1}, s_{2}-p} a_{i}\left(m-s_{1}\right) e_{l}\left(s_{2}\right) .
$$

Similarly, we have

$$
\begin{gathered}
{\left[a_{i}\left(m-s_{1}\right) a_{j}^{*}\left(s_{1}\right), a_{l}^{*}\left(s_{2}\right) e_{k}^{*}\left(p-s_{2}\right)\right]_{-}=-\delta_{i l} \delta_{m-s_{1},-s_{2}} a_{j}^{*}\left(s_{1}\right) e_{k}^{*}\left(p-s_{2}\right),} \\
{\left[e_{j}\left(s_{1}\right) e_{i}^{*}\left(m-s_{1}\right), a_{k}\left(p-s_{2}\right) e_{l}\left(s_{2}\right)\right]_{-}=-\delta_{i l} \delta_{m-s_{1},-s_{2}} e_{j}\left(s_{1}\right) a_{k}\left(p-s_{2}\right),} \\
{\left[e_{j}\left(s_{1}\right) e_{i}^{*}\left(m-s_{1}\right), a_{l}^{*}\left(s_{2}\right) e_{k}^{*}\left(p-s_{2}\right)\right]_{-}=\delta_{j k} \delta_{s_{1}, s_{2}-p} a_{l}^{*}\left(s_{2}\right) e_{i}^{*}\left(m-s_{1}\right) .}
\end{gathered}
$$


Then we replace $s_{1}$ or $s_{2}$ in the above four terms by $s$ :

$$
\begin{aligned}
& {\left[g_{i j}(m, n), h_{k l}(p, t)\right]_{-i}} \\
& \qquad \begin{array}{l}
\delta_{j k} q^{n p} \sum_{s \in Z} q^{-(n+t) s}\left(a_{i}(m+p-s) e_{l}(s)+a_{l}^{*}(s) e_{i}^{*}(m+p-s)\right) \\
\quad-\delta_{i l} q^{t m} \sum_{s \in Z} q^{-(n+t) s}\left(a_{k}(m+p-s) e_{j}(s)+a_{j}^{*}(s) e_{k}^{*}(m+p-s)\right)
\end{array} \\
& \quad \delta_{j k} q^{n p} h_{i l}(m+p, n+t)-\delta_{i l} q^{t m} h_{k j}(m+p, n+t) .
\end{aligned}
$$

\section{Lemma 3.6.}

$\left[g_{i j}(m, n), g_{k l}(p, t)\right]_{-}=\delta_{j k} q^{n p} g_{i l}(m+p, n+t)-\delta_{i l} q^{t m} g_{k j}(m+p, n+t)$.

Proof.

$$
\begin{array}{r}
{\left[g_{i j}(m, n), g_{k l}(p, t)\right]_{-}=\sum_{s_{1}, s_{2} \in Z} q^{-n s_{1}-t s_{2}}\left[a_{i}\left(m-s_{1}\right) a_{j}^{*}\left(s_{1}\right)+e_{j}\left(s_{1}\right) e_{i}^{*}\left(m-s_{1}\right),\right.} \\
\left.a_{k}\left(p-s_{2}\right) a_{l}^{*}\left(s_{2}\right)+e_{l}\left(s_{2}\right) e_{k}^{*}\left(p-s_{2}\right)\right]_{-} .
\end{array}
$$

Then, for $\left[a_{i}\left(m-s_{1}\right) a_{j}^{*}\left(s_{1}\right), a_{k}\left(p-s_{2}\right) a_{l}^{*}\left(s_{2}\right)\right]_{-}$, by using Proposition 3.1 we have

$$
\begin{aligned}
{\left[a_{i}\left(m-s_{1}\right) a_{j}^{*}\left(s_{1}\right)\right.} & \left., a_{k}\left(p-s_{2}\right) a_{l}^{*}\left(s_{2}\right)\right]_{-} \\
& =-\delta_{i l} \delta_{m-s_{1},-s_{2}} a_{k}\left(p-s_{2}\right) a_{j}^{*}\left(s_{1}\right)+\delta_{j k} \delta_{s_{1}, s_{2}-p} a_{i}\left(m-s_{1}\right) a_{l}^{*}\left(s_{2}\right) .
\end{aligned}
$$

Using Proposition 3.2,

$$
\begin{aligned}
& {\left[e_{j}\left(s_{1}\right) e_{i}^{*}\left(m-s_{1}\right), e_{l}\left(s_{2}\right) e_{k}^{*}\left(p-s_{2}\right)\right]_{-}} \\
& \quad=-\delta_{i l} \delta_{m-s_{1},-s_{2}} e_{j}\left(s_{1}\right) e_{k}^{*}\left(p-s_{2}\right)+\delta_{j k} \delta_{s_{1}, s_{2}-p} e_{l}\left(s_{2}\right) e_{i}^{*}\left(m-s_{1}\right) .
\end{aligned}
$$

Clearly,

$\left[a_{i}\left(m-s_{1}\right) a_{j}^{*}\left(s_{1}\right), e_{l}\left(s_{2}\right) e_{k}^{*}\left(p-s_{2}\right)\right]_{-}=\left[e_{j}\left(s_{1}\right) e_{i}^{*}\left(m-s_{1}\right), a_{k}\left(p-s_{2}\right) a_{l}^{*}\left(s_{2}\right)\right]_{-}=0$.

From (3-3) and (3-2), we have

$$
\begin{aligned}
a_{k}\left(p-s_{2}\right) a_{j}^{*}\left(s_{1}\right) & =: a_{k}\left(p-s_{2}\right) a_{j}^{*}\left(s_{1}\right):+\delta_{j k} \delta_{s_{1}, s_{2}-p} \theta\left(p-s_{1}-s_{2}\right), \\
e_{j}\left(s_{1}\right) e_{k}^{*}\left(p-s_{2}\right) & =: e_{j}\left(s_{1}\right) e_{k}^{*}\left(p-s_{2}\right):+\delta_{j k} \delta_{s_{1}, s_{2}-p} \theta\left(s_{1}+s_{2}-p\right), \\
a_{i}\left(m-s_{1}\right) a_{l}^{*}\left(s_{2}\right) & =: a_{i}\left(m-s_{1}\right) a_{l}^{*}\left(s_{2}\right):+\delta_{i l} \delta_{m-s_{1},-s_{2}} \theta\left(m-s_{1}-s_{2}\right), \\
e_{l}\left(s_{2}\right) e_{i}^{*}\left(m-s_{1}\right) & =: e_{l}\left(s_{2}\right) e_{i}^{*}\left(m-s_{1}\right):+\delta_{i l} \delta_{m-s_{1},-s_{2}} \theta\left(s_{1}+s_{2}-m\right), \\
\theta\left(p-s_{1}-s_{2}\right)+\theta\left(s_{1}+s_{2}-p\right) & =\theta\left(m-s_{1}-s_{2}\right)+\theta\left(s_{1}+s_{2}-m\right)=1 .
\end{aligned}
$$


So

$$
\begin{aligned}
-\delta_{i l} \delta_{m-s_{1},-s_{2}} a_{k}\left(p-s_{2}\right) a_{j}^{*}\left(s_{1}\right)+\delta_{j k} \delta_{s_{1}, s_{2}-p} a_{i}\left(m-s_{1}\right) a_{l}^{*}\left(s_{2}\right) & \\
-\delta_{i l} \delta_{m-s_{1},-s_{2}} e_{j}\left(s_{1}\right) e_{k}^{*}\left(p-s_{2}\right)+\delta_{j k} \delta_{s_{1}, s_{2}-p} e_{l}\left(s_{2}\right) e_{i}^{*}\left(m-s_{1}\right) & \\
= & \delta_{j k} \delta_{s_{1}, s_{2}-p}\left(: a_{i}\left(m-s_{1}\right) a_{l}^{*}\left(s_{2}\right):+: e_{l}\left(s_{2}\right) e_{i}^{*}\left(m-s_{1}\right):\right) \\
& \quad-\delta_{i l} \delta_{m-s_{1},-s_{2}}\left(: a_{k}\left(p-s_{2}\right) a_{j}^{*}\left(s_{1}\right):+: e_{j}\left(s_{1}\right) e_{k}^{*}\left(p-s_{2}\right):\right) .
\end{aligned}
$$

Then we get

$$
\begin{aligned}
& {\left[g_{i j}(m, n), g_{k l}(p, t)\right]_{-}} \\
& =\sum_{s_{1}, s_{2} \in Z} q^{-n s_{1}-t s_{2}}\left\{\delta_{j k} \delta_{s_{1}, s_{2}-p}\left(: a_{i}\left(m-s_{1}\right) a_{l}^{*}\left(s_{2}\right):+: e_{l}\left(s_{2}\right) e_{i}^{*}\left(m-s_{1}\right):\right)\right. \\
& \left.-\delta_{i l} \delta_{m-s_{1},-s_{2}}\left(: a_{k}\left(p-s_{2}\right) a_{j}^{*}\left(s_{1}\right):+e_{j}\left(s_{1}\right) e_{k}^{*}\left(p-s_{2}\right):\right)\right\} .
\end{aligned}
$$

Now we replace $s_{1}$ or $s_{2}$ in the above terms by $s$; we get $\left[g_{i j}(m, n), g_{k l}(p, t)\right]_{-}$

$$
\begin{aligned}
= & \delta_{j k} q^{n p} \sum_{s \in Z} q^{-(n+t) s}\left(a_{i}(m+p-s) a_{l}^{*}(s)+e_{l}(s) e_{i}^{*}(m+p-s)\right) \\
& \quad-\delta_{i l} q^{t m} \sum_{s \in Z} q^{-(n+t) s}\left(a_{k}(m+p-s) a_{j}^{*}(s)+e_{j}(s) e_{k}^{*}(m+p-s)\right) \\
= & \delta_{j k} q^{n p} g_{i l}(m+p, n+t)-\delta_{i l} q^{t m} g_{k j}(m+p, n+t) .
\end{aligned}
$$

Although $g_{i j}(m, n)$ and $h_{i j}(m, n)$ are infinite sums, they are well defined as operators on $V(N)$ since at most finitely many terms can have a nontrivial action on any $v \in V(N)=\alpha(N,+1) \otimes \alpha(N,-1) v_{0}^{+} \otimes v_{0}^{-}$.

Then from Lemmas 3.4, 3.5 and 3.6 we have:

Theorem 3.7. $V(N)$ is a module for the $Q(N-1)$-graded Lie superalgebra $\mathcal{Q}$ under the action given by

$$
\begin{aligned}
& \pi\left(\tilde{g}_{i j}(m, n)\right)=g_{i j}(m, n), \\
& \pi\left(\tilde{h}_{i j}(m, n)\right)=h_{i j}(m, n),
\end{aligned}
$$

for all $m, n \in \mathbb{Z}$ and $1 \leq i, j \leq N$.

\section{References}

[Allison et al. 1997] B. N. Allison, S. Azam, S. Berman, Y. Gao, and A. Pianzola, Extended affine Lie algebras and their root systems, Mem. Amer. Math. Soc. 603, American Mathematical Society, Providence, RI, 1997. MR Zbl

[Allison et al. 2000] B. Allison, G. Benkart, and Y. Gao, "Central extensions of Lie algebras graded by finite root systems”, Math. Ann. 316:3 (2000), 499-527. MR Zbl 
[Benkart and Zelmanov 1996] G. Benkart and E. Zelmanov, "Lie algebras graded by finite root systems and intersection matrix algebras”, Invent. Math. 126:1 (1996), 1-45. MR Zbl

[Berman and Moody 1992] S. Berman and R. V. Moody, "Lie algebras graded by finite root systems and the intersection matrix algebras of Slodowy", Invent. Math. 108:2 (1992), 323-347. MR Zbl

[Berman et al. 1996] S. Berman, Y. Gao, and Y. S. Krylyuk, "Quantum tori and the structure of elliptic quasi-simple Lie algebras”, J. Funct. Anal. 135:2 (1996), 339-389. MR Zbl

[Chen and Gao 2007] H. Chen and Y. Gao, " $B C_{N}$-graded Lie algebras arising from fermionic representations", J. Algebra 308:2 (2007), 545-566. MR Zbl

[Chen et al. 2006] H. Chen, Y. Gao, and S. Shang, " $B(0, N)$-graded Lie superalgebras coordinatized by quantum tori”, Sci. China Ser. A 49:11 (2006), 1740-1752. MR Zbl

[Feingold and Frenkel 1985] A. J. Feingold and I. B. Frenkel, "Classical affine algebras", Adv. in Math. 56:2 (1985), 117-172. MR Zbl

[Frenkel 1980] I. B. Frenkel, "Spinor representations of affine Lie algebras", Proc. Nat. Acad. Sci. U.S.A. 77:11 (1980), 6303-6306. MR Zbl

[Gao 2002] Y. Gao, "Fermionic and bosonic representations of the extended affine Lie algebra $\mathfrak{g l}_{N}\left(\mathbb{C}_{q}\right)$ ", Canad. Math. Bull. 45:4 (2002), 623-633. MR Zbl

[Kac 1977] V. G. Kac, "Lie superalgebras”, Advances in Math. 26:1 (1977), 8-96. MR Zbl

[Kac and Peterson 1981] V. G. Kac and D. H. Peterson, "Spin and wedge representations of infinitedimensional Lie algebras and groups", Proc. Nat. Acad. Sci. U.S.A. 78:6, part 1 (1981), 3308-3312. MR Zbl

[Martínez and Zelmanov 2003] C. Martínez and E. I. Zelmanov, "Lie superalgebras graded by $P(n)$ and Q(n)", Proc. Natl. Acad. Sci. USA 100:14 (2003), 8130-8137. MR Zbl

Received August 29, 2015. Revised December 20, 2015.

\section{JIN CHENG}

DEPARTMENT OF MATHEMATICS

University of SCIENCE AND TECHNOLOGY OF CHINA

HEFEI, 230026

CHINA

cheng934@mail.ustc.edu.cn 


\title{
PACIFIC JOURNAL OF MATHEMATICS
}

Founded in 1951 by E. F. Beckenbach (1906-1982) and F. Wolf (1904-1989)

$$
\text { msp.org/pjm }
$$

\section{EDITORS}

\author{
Don Blasius (Managing Editor) \\ Department of Mathematics \\ University of California \\ Los Angeles, CA 90095-1555 \\ blasius@math.ucla.edu
}

\author{
Paul Balmer \\ Department of Mathematics \\ University of California \\ Los Angeles, CA 90095-1555 \\ balmer@math.ucla.edu \\ Robert Finn \\ Department of Mathematics \\ Stanford University \\ Stanford, CA 94305-2125 \\ finn@math.stanford.edu \\ Sorin Popa \\ Department of Mathematics \\ University of California \\ Los Angeles, CA 90095-1555 \\ popa@math.ucla.edu
}

\author{
Vyjayanthi Chari \\ Department of Mathematics \\ University of California \\ Riverside, CA 92521-0135 \\ chari@math.ucr.edu \\ Kefeng Liu \\ Department of Mathematics \\ University of California \\ Los Angeles, CA 90095-1555 \\ liu@math.ucla.edu \\ Igor Pak \\ Department of Mathematics \\ University of California \\ Los Angeles, CA 90095-1555 \\ pak.pjm@gmail.com \\ Paul Yang \\ Department of Mathematics \\ Princeton University \\ Princeton NJ 08544-1000 \\ yang@math.princeton.edu
}

\section{PRODUCTION}

Silvio Levy, Scientific Editor, production@msp.org

\section{SUPPORTING INSTITUTIONS}

ACADEMIA SINICA, TAIPEI

CALIFORNIA INST. OF TECHNOLOGY

STANFORD UNIVERSITY

UNIV. OF BRITISH COLUMBIA

UNIV. OF CALIFORNIA, BERKELEY

UNIV. OF CALIFORNIA, DAVIS

UNIV. OF CALIFORNIA, LOS ANGELES

UNIV. OF CALIFORNIA, RIVERSIDE

UNIV. OF CALIFORNIA, SAN DIEGO

UNIV. OF CALIF., SANTA BARBARA
KEIO UNIVERSITY

MATH. SCIENCES RESEARCH INSTITUTE

NEW MEXICO STATE UNIV.

OREGON STATE UNIV.
Daryl Cooper

Department of Mathematics

University of California

Santa Barbara, CA 93106-3080 cooper@math.ucsb.edu

Jiang-Hua Lu

Department of Mathematics

The University of Hong Kong

Pokfulam Rd., Hong Kong

jhlu@maths.hku.hk

$$
\text { Jie Qing }
$$

Department of Mathematics

University of California

Santa Cruz, CA 95064

qing@cats.ucsc.edu

\author{
UNIV. OF CALIF., SANTA CRUZ \\ UNIV. OF MONTANA \\ UNIV. OF OREGON \\ UNIV. OF SOUTHERN CALIFORNIA \\ UNIV. OF UTAH \\ UNIV. OF WASHINGTON \\ WASHINGTON STATE UNIVERSITY
}

These supporting institutions contribute to the cost of publication of this Journal, but they are not owners or publishers and have no responsibility for its contents or policies.

See inside back cover or msp.org/pjm for submission instructions.

The subscription price for 2016 is US \$/year for the electronic version, and \$/year for print and electronic.

Subscriptions, requests for back issues and changes of subscriber address should be sent to Pacific Journal of Mathematics, P.O. Box 4163, Berkeley, CA 94704-0163, U.S.A. The Pacific Journal of Mathematics is indexed by Mathematical Reviews, Zentralblatt MATH, PASCAL CNRS Index, Referativnyi Zhurnal, Current Mathematical Publications and Web of Knowledge (Science Citation Index).

The Pacific Journal of Mathematics (ISSN 0030-8730) at the University of California, c/o Department of Mathematics, 798 Evans Hall \#3840, Berkeley, CA 94720-3840, is published twelve times a year. Periodical rate postage paid at Berkeley, CA 94704, and additional mailing offices. POSTMASTER: send address changes to Pacific Journal of Mathematics, P.O. Box 4163, Berkeley, CA 94704-0163.

PJM peer review and production are managed by EditFLOW ${ }^{\circledR}$ from Mathematical Sciences Publishers.

PUBLISHED BY

\section{I. mathematical sciences publishers}

nonprofit scientific publishing

http://msp.org/

(C) 2016 Mathematical Sciences Publishers 


\section{PACIFIC JOURNAL OF MATHEMATICS}

Volume $283 \quad$ No. $1 \quad$ July 2016

A New family of simple $\mathfrak{g l}_{2 n}(\mathbb{C})$-modules

JONATHAN NILSSON

Derived categories of representations of small categories over commutative noetherian rings

BENJAMIN ANTIEAU and GREg STEVENSON

Vector bundles over a real elliptic curve

INDRANIL BISWAS and FLORENT SCHAFFHAUSER

$\mathrm{Q}(\mathrm{N})$-graded Lie superalgebras arising from fermionic-bosonic representations

JIN CHENG

Conjugacy and element-conjugacy of homomorphisms of compact Lie groups

YiNGJUE FANG, GANG HAN and BINYONG SUN

Entire sign-changing solutions with finite energy to the fractional Yamabe equation

DANILO GARRIDO and MoniCa Musso

Calculation of local formal Mellin transforms

ADAM GRAHAM-SQUIRE

The untwisting number of a knot

KENAN INCE

A Plancherel formula for $L^{2}(G / H)$ for almost symmetric subgroups

Bent Ørsted and Birgit SPEH

Multiplicative reduction and the cyclotomic main conjecture for $\mathrm{GL}_{2}$

CHRISTOPHER SKINNER

Commensurators of solvable $S$-arithmetic groups

\section{DANIEL STUDENMUND}

Gerstenhaber brackets on Hochschild cohomology of quantum symmetric algebras and their group extensions

SARAH WITHERSPOON and GUODONG ZHOU 